Nonlin. Processes Geophys., 28, 111-119, 2021

https://doi.org/10.5194/npg-28-111-2021

(C) Author(s) 2021. This work is distributed under

the Creative Commons Attribution 4.0 License.

\title{
Training a convolutional neural network to conserve mass in data assimilation
}

\author{
Yvonne Ruckstuhl ${ }^{1}$, Tijana Janjić ${ }^{1}$, and Stephan Rasp ${ }^{2}$ \\ ${ }^{1}$ Meteorological Institute Munich, Ludwig-Maximilians-Universität München, Munich, Germany \\ ${ }^{2}$ ClimateAi, San Francisco, CA, USA
}

Correspondence: Yvonne Ruckstuhl (yvonne.ruckstuhl@lmu.de)

Received: 2 September 2020 - Discussion started: 25 September 2020

Revised: 15 December 2020 - Accepted: 17 December 2020 - Published: 9 February 2021

\begin{abstract}
In previous work, it was shown that the preservation of physical properties in the data assimilation framework can significantly reduce forecast errors. Proposed data assimilation methods, such as the quadratic programming ensemble (QPEns) that can impose such constraints on the calculation of the analysis, are computationally more expensive, severely limiting their application to high-dimensional prediction systems as found in Earth sciences. We, therefore, propose using a convolutional neural network $(\mathrm{CNN})$ trained on the difference between the analysis produced by a standard ensemble Kalman filter (EnKF) and the QPEns to correct any violations of imposed constraints. In this paper, we focus on the conservation of mass and show that, in an idealised set-up, the hybrid of a CNN and the EnKF is capable of reducing analysis and background errors to the same level as the QPEns.
\end{abstract}

\section{Introduction}

The ensemble Kalman filter (EnKF; Evensen, 1994; Burgers et al., 1998; Evensen, 2009) and versions thereof are powerful data assimilation algorithms that can be applied to problems that need an estimate of a high-dimensional model state, as in weather forecasting. An important condition for the successful application of the EnKF to a large system is the use of localisation. Any localisation method aims to diminish sampling errors caused by the computational limitation of the ensemble size. By doing so, mass conservation, as guaranteed by a numerical model, is violated during data assimilation (Janjić et al., 2014). It was shown in Janjić et al. (2014), Zeng and Janjić (2016), Zeng et al. (2017) and Ruckstuhl and Janjić (2018) that failing to conserve certain quantities like mass, energy and enstrophy can be highly detrimental to the estimation of the state. Janjić et al. (2014) proposed a new data assimilation algorithm, the quadratic programming ensemble (QPEns), which replaces the analysis equations of the EnKF with an ensemble of minimisation problems subject to physical constraints. Zeng et al. (2017) showed, in an idealised set-up with a 2 week forecast generated by a $2 \mathrm{D}$ shallow water model, that error growth is significantly reduced if the enstrophy is constrained. Similarly, Ruckstuhl and Janjić (2018) illustrated the benefit of constraining the total mass and positivity of precipitation in a simple test case for convective-scale data assimilation. The obstacle that remains in applying the QPEns on large systems is the computational demand of solving the constrained minimisation problems that appear for each ensemble member at each assimilation cycle. For a detailed discussion on the computational costs of the QPEns, we refer to Janjic et al. (2021). In this work, we propose using an artificial neural network (NN) to correct the unconstrained solution instead of solving the constrained minimisation problems.

NNs are powerful tools to approximate arbitrary nonlinear functions (Nielsen, 2015). A NN learns to recognise patterns based on examples, rather than being explicitly programmed. An important advantage is that no direct knowledge of the function is needed. Instead, a data set consisting of inputoutput pairs is used to train the NN to predict the output corresponding to a given input. Especially in the fields of image recognition and natural language processing, NNs are state of the art and have become a standard tool (LeCun Yann et al., 2015). In numerical weather prediction, NNs are not yet fully integrated, though interest is rising quickly (Reichstein et al., 
2019). A recent review of the use of NNs in meteorology can be found in McGovern et al. (2019). Explored applications include (but are not limited to) post-processing of raw model output based on observations (McGovern et al., 2017; Rasp and Lerch, 2021), representing subgrid processes in weather and climate models using high-resolution model simulations (Krasnopolsky et al., 2013; Rasp et al., 2018; Brenowitz and Bretherton, 2019; Yuval and O'Gorman, 2020), combining a NN with a knowledge-based model as a hybrid forecasting approach (Pathak et al., 2018b; Watson, 2019) and replacing the numerical weather prediction model altogether (Dueben and Bauer, 2018; Pathak et al., 2018a; Weyn et al., 2020; Scher and Messori, 2019; Rasp et al., 2020; Rasp and Thuerey, 2020). A general challenge when applying NNs in numerical weather prediction is that the training data often consist of sparse and noisy data, which NNs are ill equipped to handle. Brajard et al. (2020a) and Bocquet et al. (2020) proposed using data assimilation in the training process of the NN to deal with this issue. This approach has been successfully applied to reduce model errors (Brajard et al., 2020b; Farchi et al., 2020).

Fully replacing data assimilation by a $\mathrm{NN}$ has been attempted by Cintra and de Campos Velho (2014) in the context of a simplified atmospheric general circulation model. They trained on a cycling data set produced by the local ensemble transform Kalman filter (LETKF; Bishop et al., 2001; Hunt et al., 2007) and showed that the trained NN performs nearly as well as the LETKF, with significantly reduced computational effort. Other applications of NNs in the context of data assimilation are for observational bias correction (Jin et al., 2019) and tuning of covariance localisation (Moosavi et al., 2019). In this paper, we take an approach that, by combining the NN with a data assimilation algorithm, will allow the extraction of the most information from sparse and noisy observations, as argued in, for example, Brajard et al. (2020a). We aim to produce better results than standard data assimilation algorithms at minimal additional computational costs by training on data produced by the QPEns.

We generate our training data by performing twin experiments with the 1D modified shallow water model (Würsch and Craig, 2014), which was designed to mimic important properties of convection. These aspects include an acute regime switch when convection is triggered (conditional instability) and a significant time lag between the onset of convection and its observation. The model is briefly introduced in Sect. 2.1, followed by the settings of the twin experiments in Sect. 2.2. Section 2.3 provides a report on the generation of the training data. Since both our input and output are full model states, the obvious choice is to train a convolutional neural network $(\mathrm{CNN})$, as the convolution with kernels naturally acts as a form of localisation. The CNN architecture we use for this application is described in Sect. 2.4. The results are presented in Sect. 3, followed by the conclusion in Sect. 4.

\section{Experiment set-up}

\subsection{Model}

The modified shallow water model (Würsch and Craig, 2014) consists of the following equations for the velocity $u$, rain $r$ and water height level of the fluid $h$, respectively:

$$
\frac{\partial u}{\partial t}+u \frac{\partial u}{\partial x}+\frac{\partial\left(\phi+\gamma^{2} r\right)}{\partial x}=\beta_{u}+D_{u} \frac{\partial^{2} u}{\partial x^{2}},
$$

with the following:

$$
\begin{aligned}
& \phi= \begin{cases}\phi_{\mathrm{c}} & \text { if } h>h_{\mathrm{c}} \\
g h & \text { else, }\end{cases} \\
& \frac{\partial r}{\partial t}+u \frac{\partial r}{\partial x}=D_{r} \frac{\partial^{2} r}{\partial x^{2}}-\alpha r \\
& - \begin{cases}\delta \frac{\partial u}{\partial x}, & h>h_{r} \quad \text { and } \quad \frac{\partial u}{\partial x}<0 \\
0, & \text { else, }\end{cases} \\
& \frac{\partial h}{\partial t}+\frac{\partial(u h)}{\partial x}=D_{h} \frac{\partial^{2} h}{\partial x^{2}} .
\end{aligned}
$$

Above, $h_{\mathrm{c}}$ represents the level of free convection. When this threshold is reached, the geopotential $\phi$ takes on a lower constant value $\phi_{\mathrm{c}}$. The parameters $D_{u}, D_{r}$ and $D_{h}$ are the diffusion constants corresponding to $u, r$ and $h$, respectively. The coefficient $\gamma:=\sqrt{g h_{0}}$ is the gravity wave speed for the absolute fluid layer $h_{0}\left(h_{0}<h_{\mathrm{c}}\right)$. The small Gaussian-shaped forcing $\beta_{u}$ is added at random locations to the velocity $u$ at every model time step. This is done in order to trigger perturbations that lead to convection. Parameters $\delta$ and $\alpha$ are the production and removal rate of rain, respectively. When $h$ reaches the rain threshold $h_{r}\left(h_{r}>h_{\mathrm{c}}\right)$, rain is produced, leading to a decrease in the water level and buoyancy. The model conserves mass, so the spatial integral over $h$ is constant in time.

The $1 \mathrm{D}$ model domain, representing $125 \mathrm{~km}$, is discretised with $n=250$ points, yielding the state vector $\boldsymbol{x}=$ $\left[\boldsymbol{u}^{T} \boldsymbol{h}^{T} \boldsymbol{r}^{T}\right]^{T} \in \mathbb{R}^{750}$. The time step is chosen to be $5 \mathrm{~s}$. The forcing $\beta_{u}$ has a Gaussian shape, with a half-width of four grid points and an amplitude of $0.002 \mathrm{~m} \mathrm{~s}^{-1}$. This model was used for testing data assimilation methods in convectivescale applications in Haslehner et al. (2016) and Ruckstuhl and Janjić (2018).

\subsection{Twin experiments}

The nature run which mimics the true state of the atmosphere is a model simulation starting from an arbitrary initial state. The ensemble is chosen to be of a small size with $N_{\text {ens }}=10$, and, like the nature run, each member starts from an arbitrary initial state. Observations are assimilated at every $\mathrm{dT}$ model time step and are obtained by adding a Gaussian error to the wind $u$ and height $h$ field of the nature run at the corresponding time, with a standard deviation of $\sigma_{u}=0.001 \mathrm{~m} \mathrm{~s}^{-1}$ and 
$\sigma_{h}=0.01 \mathrm{~m}$, and a log-normal error is added to the rain $r$ field with the parameters of the underlying normal distribution $\mu=-8$ and $\sigma=1.5$. For all variables, the observation error is roughly $10 \%$ of the maximum deviation from the variable mean. To mimic radar data, observations for all variables are available only on grid points where rain above a threshold of $0.005 \mathrm{dBZ}$ is measured. A random selection, amounting to $10 \%$ of the remaining grid points of additional wind observations, are assimilated, which represents additional available data (for example, obtained from aircraft).

To deal with undersampling, covariance localisation using the fifth piecewise rational function (Gaspari and Cohn, 1999 ) is applied with a localisation radius of four grid points. This corresponds to the localisation radius for which the EnKF yields minimum analysis root mean squared error (RMSE) values of the rain variable for an ensemble size of 10. An interior point method is used to solve the quadratic minimisation problems of the QPEns. The constraints that are applied are mass conservation, i.e. $\boldsymbol{e}^{T}\left(\boldsymbol{h}^{\mathrm{a}}-\boldsymbol{h}^{\mathrm{b}}\right)=\boldsymbol{e}^{T} \boldsymbol{\delta} \boldsymbol{h}=$ 0 , and positivity of precipitation, i.e. $\boldsymbol{r}^{\mathrm{a}}=\boldsymbol{\delta} \boldsymbol{r}+\boldsymbol{r}^{\mathrm{b}} \geq 0$. Here, the superscript $\mathrm{b}$ denotes the background and a the analysis, and $\boldsymbol{e}$ is a vector of size $n$ containing only values of one. For the EnKF, negative values for rain are set to zero if they occur.

When the assimilation window $\mathrm{dT}$ is large enough, the accumulation of mass leads to divergence for the EnKF; that is, the analysis error is larger than the climatological standard deviation of the model state. The QPEns converges for all dT, due to its ability to conserve mass. We, therefore, distinguish between two cases, namely one where the EnKF converges $(\mathrm{dT}=60$; equivalent to $5 \mathrm{~min}$ real time) and one where the EnKF diverges ( $\mathrm{dT}=120$; equivalent to $10 \mathrm{~min}$ real time). We refer to Ruckstuhl and Janjić (2018) for a comparison of the performance of the EnKF and the QPEns as a function of ensemble size for different localisation radii, assimilation windows and observation coverage.

\subsection{Training data}

We aim to produce initial conditions of the same quality as the ones produced by the QPEns by upgrading the initial conditions produced by the EnKF using a CNN. To that end, we generate QPEns cycling data $\left\{\left(\mathbf{Q}_{t}^{\mathrm{b}}, \mathbf{Q}_{t}^{\mathrm{a}}\right): t=1,2, \ldots, T\right\}$, where $\mathbf{Q}$ stands for QPEns and the superscripts b denote the background and a the analysis, respectively. In parallel, we create the data set $\left\{\mathbf{X}_{t}^{\mathrm{a}}: t=1,2, \ldots, T\right\}$, where $\mathbf{X}_{t}^{\mathrm{a}}$ is the unconstrained solution calculated from $\mathbf{Q}_{t}^{\mathrm{b}}$. See Fig. 1 for a schematic of the generation process of the data sets. Note that, by using the background generated from the QPEns $\left(\mathbf{Q}_{t}^{\mathrm{b}}\right)$ in the calculation of both $\mathbf{X}_{t}^{\mathrm{a}}$ and $\mathbf{Q}_{t}^{\mathrm{a}}$, we train the CNN only to focus on differences in the minimisation process and not on the possible differences in the background error covariances that could have accumulated during cycling. In Sect. 3, we validate this approach by applying the CNN to the EnKF analysis for 180 subsequent data assimilation cy-

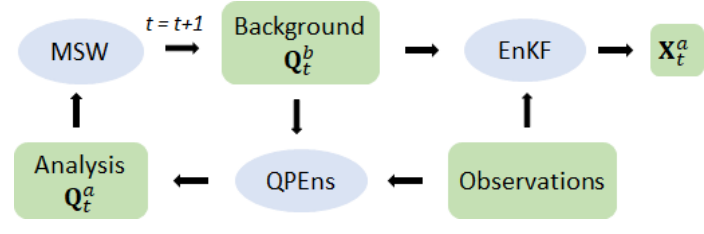

Figure 1. Schematic of the generation of the data sets $\mathbf{Q}_{t}^{\mathrm{b}}, \mathbf{Q}_{t}^{\mathrm{a}}$ and $\mathbf{X}_{t}^{\mathrm{a}}$, where MSW stands for modified shallow water model.
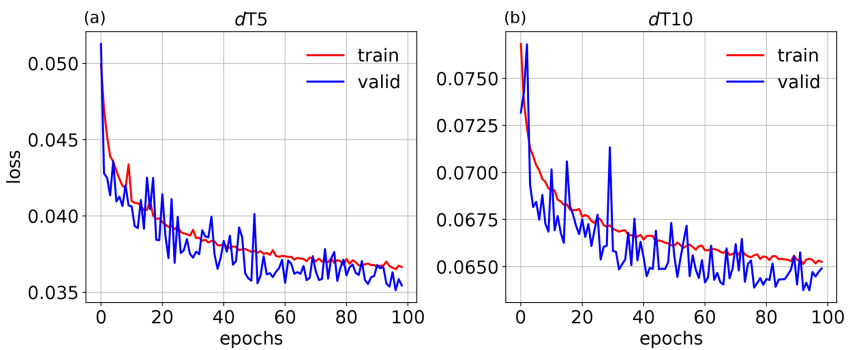

Figure 2. Value of the loss function $J$ averaged over samples for the training (red) and validation (blue) data set as a function of epochs for dT5 (a) and dT10 (b).

cles. Both data sets contain the entire ensemble of $N_{\text {ens }}=10$ members, such that $(*)_{t}^{(*)} \in \mathbb{R}^{N_{\text {ens }} \times n \times 3}$, where the last dimension represents the three variables $(u, h$ and $r)$, and $n$ is the number of grid points.

The output of our training set $\mathbf{Y}^{\mathrm{tr}} \in \mathbb{R}^{N_{\mathrm{ens}} T \times n \times 3}$ is simply a reshaped and normalised version of the data set $\left\{\mathbf{Q}_{t}^{\mathrm{a}}\right.$ : $t=1,2, \ldots, T\}$. For the input of our training set $\mathbf{X}^{\mathrm{tr}}$, we choose to use an index vector indicating the position of the radar observations $\left\{\mathbf{I}_{t}: t=1,2, \ldots, T\right\}$, in addition to the unconstrained solutions $\left\{\mathbf{X}_{t}^{\mathrm{a}}: t=1,2, \ldots, T\right\}$, yielding $\mathbf{X}^{\operatorname{tr}} \in$ $\mathbb{R}^{N_{\text {ens }} T \times n \times 4}$, where the index vector $\mathbf{I}_{t}$ is copied $N_{\text {ens }}$ times to obtain $\mathbf{I}_{t}^{*} \in \mathbb{R}^{N_{\text {ens }} \times n \times 1}$. We include this information because we know from Ruckstuhl and Janjić (2018) that the strength of the QPEns lies in suppressing spurious convection. Since the radar observations cover only rainy regions, the data set $\mathbf{I}_{t}$ can help the CNN to distinguish between dry and rainy regions and possibly develop a different regime for each situation. We verified that the $\mathrm{CNN}$ yields significantly different output when setting all values of $\mathbf{I}_{t}$ to zero, indicating that the CNN indeed uses this information. For $u$ and $h$, the input and output data set is normalised by subtracting the climatological mean before dividing by the climatological standard deviation. For $r$, we do not subtract the climatological mean to maintain positivity.

A validation data set $\mathbf{X}^{\text {valid }}$ and $\mathbf{Y}^{\text {valid }}$ is exactly the same as the training data set, but a different random seed number is created to monitor the training process. For both the training and validation data set, we set $T=4800$, which amounts to a total of $N_{\text {ens }} T=48000$ training and validation samples, respectively. 

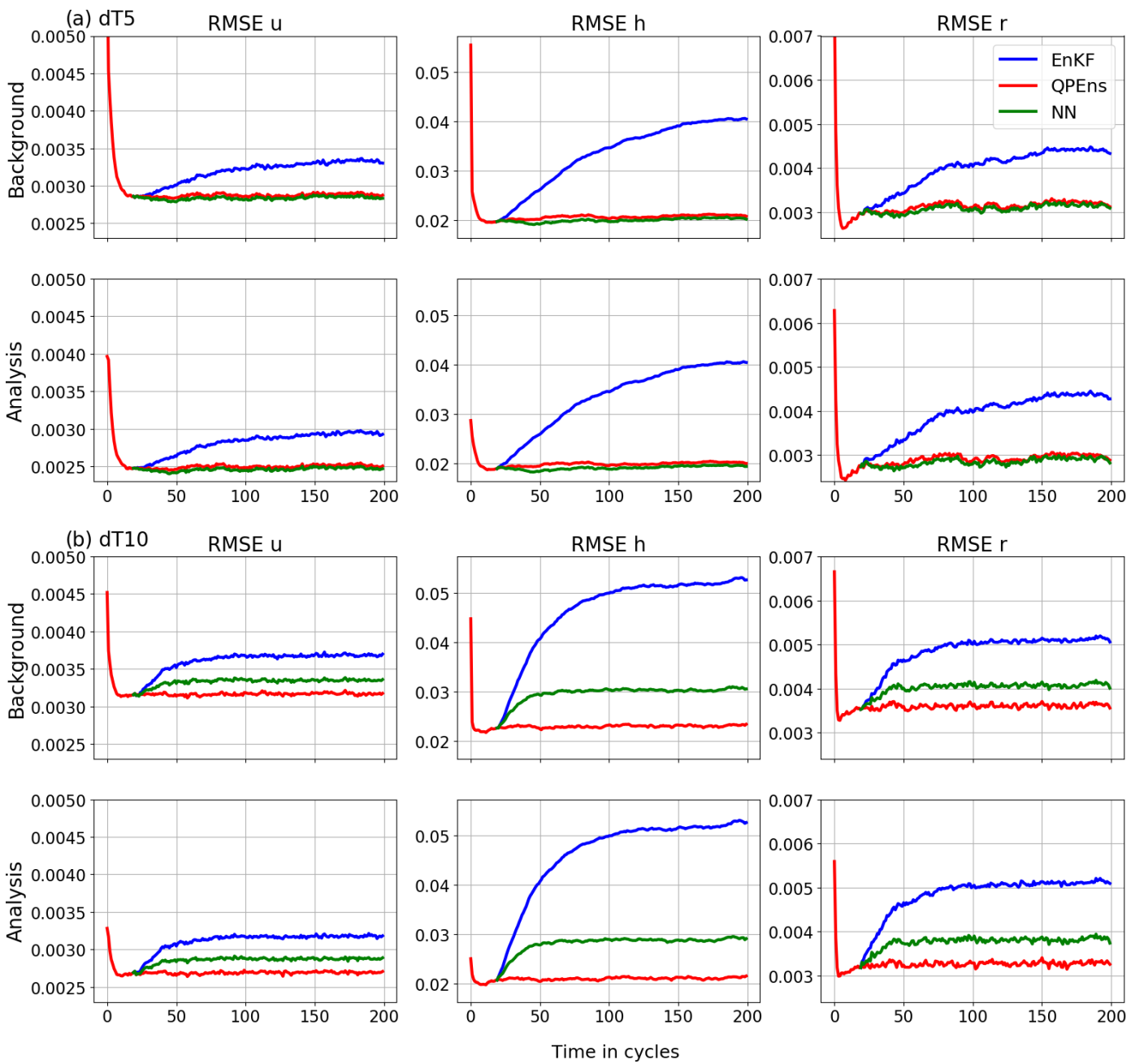

Figure 3. Root mean squared error (RMSE) of the ensemble averaged over 500 experiments of the variables (columns) for the background (top rows) and analysis (bottom rows) as functions of the assimilation cycles for the EnKF (blue), the QPEns (red) and the CNN (green). The panels in (a) correspond to dT5 and the panels in (b) to dT10.

\subsection{Convolutional neural network (CNN) architecture}

We choose to use a $\mathrm{CNN}$ with four convolutional hidden layers, consisting of 32 filters each with kernels of size 3 , and the "selu" activation function as follows:

$g(x)=\lambda_{1} \begin{cases}x, & \text { for } x \geq 0 \\ \lambda_{2}\left(e^{x}-1\right), & \text { for } x<0,\end{cases}$

where $\lambda_{1}=1.05070098$ and $\lambda_{2}=1.67326324$. These values are chosen such that the mean and variance of the inputs are preserved between two consecutive layers (Klambauer et al., 2017). The output layer is a convolutional layer as well, where the number of filters is determined by the desired shape of the output of the CNN, which is a model state $(u, h, r) \in \mathbb{R}^{n \times 3}$. The output layer has, therefore, three filters and the kernel size is again 3 . Note that the maximum influence radius of a variable as assumed by the CNN is $(3-1) / 2 \times 5=5$, where 5 is the number of layers and the kernel size is 3 . We use a linear activation function for $u$ and $h$ and the "relu" activation function for $r$ to ensure nonnegativity of rain. We set the batch size to 96 and run 100 epochs. Unless stated otherwise, the loss function is defined as the RMSE over the grid points, averaged over the variables as follows:

$J\left(\mathbf{y}_{j}^{\mathrm{p}}(\boldsymbol{w})\right)=\frac{1}{3} \sum_{v=1}^{3} \sqrt{\frac{1}{n} \sum_{i=1}^{n}\left(y_{j, i, v}^{\mathrm{p}}-y_{j, i, v}\right)^{2}}$,

$j=1, \ldots, N_{\mathrm{ens}} T$,

where $y_{j, i, v}^{\mathrm{p}}$ and $y_{j, i, v}$ are the prediction and output for the $v$ th variable of the $j$ th sample at the $i$ th grid point, respectively. The Adam algorithm is used to minimise $\frac{1}{N_{\text {ens }} T} \sum_{j=1}^{N_{\text {ens }} T} J\left(\mathbf{y}_{j}^{\mathrm{p}}(\boldsymbol{w})\right)$ over the weights $w$ of the CNN. The training is done in the Python library with Keras (Chollet, 2017). 

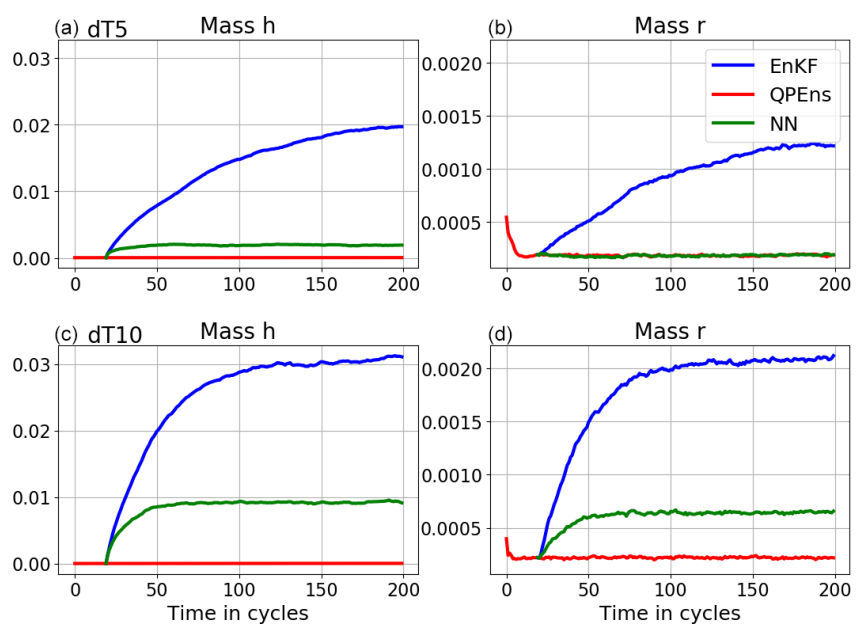

Figure 4. Absolute mass error averaged over 500 experiments of $h(\mathbf{a}, \mathbf{c})$ and $r(\mathbf{b}, \mathbf{d})$ for the analysis ensemble mean for the EnKF (blue), QPEns (red) and CNN (green). Panels (a) and (b) correspond to dT5 and panels (c) and (d) to dT10.

\section{Results}

We assign the name dT5 to the experiment corresponding to a cycling period of $5 \mathrm{~min}$ and $\mathrm{dT} 10$ to the experiment corresponding to a cycling period of $10 \mathrm{~min}$. Figure 2 shows the evolution of the loss function averaged over the samples for the training and validation data set for dT5 and dT10, respectively. Table 1 summarises what the $\mathrm{CNN}$ has learnt for each variable separately in the two cases. As the training data is normalised, we can conclude from the RMSE of the input data with respect to the output data (first row in Table 1) that the mass constraint on $h$ and the positivity constraints on $r$ impact the solution of the minimisation problem for all variables with the same order of magnitude. Given our choice of loss function, it is not surprising that the relative reduction of the gap between the input and output by the CNN is proportional to the size of the gap. By aiming to minimise the mean RMSE of all variables, the CNN reduced the violation of the mass constraint by about $20 \%$ for both experiments. However, for dT5 the reduction in the bias of the height field is $100 \%$, while for dT10 it is a mere $30 \%$.

Next, we are interested in how the CNNs perform when applied within the data assimilation cycling. In Fig. 3, we compare the performance of the EnKF, QPEns and the hybrid of $\mathrm{CNN}$ and EnKF, where CNN is applied as a correction to the initial conditions computed by the EnKF. To avoid having to train a CNN for the spin-up phase where the increments are larger, we start the data assimilation for the EnKF and the CNN from the initial conditions produced by the QPEns at the 20th cycle. The RMSEs shown in Fig. 3 are calculated through time against the nature run for both the background and the analysis.
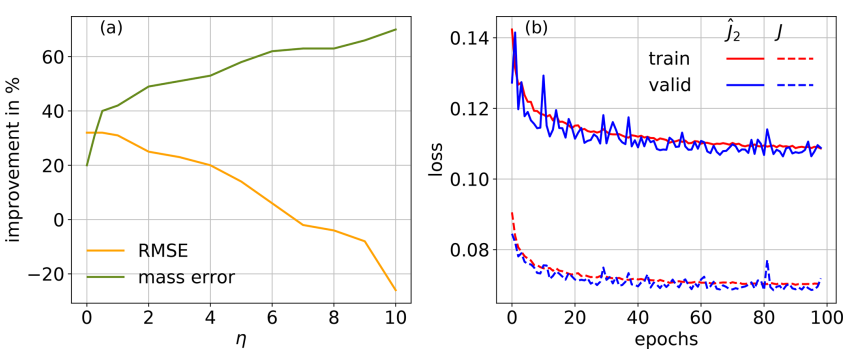

Figure 5. (a) Relative improvement in the percent of RMSE (orange) and mass error (green) towards the output with respect to the input as a function $\eta$. (b) Value of the loss function $\hat{J}$ (solid) and $J$ (dashed) averaged over samples for the training (red) and validation (blue) data set as function of epochs for $\mathrm{dT}^{1} 0_{\eta=2}$.

With respect to RMSEs, for dT5 the CNN performs as well as the QPEns, despite having learnt during training only $27 \%$ of the difference between the EnKF and QPEns analysis in terms of the loss function. For dT10 the CNN does perform significantly better than the EnKF but clearly remains inferior to the QPEns. Given that, in terms of the RMSE over the grid points, the $\mathrm{CNN}$ for dT10 is slightly better than the one for dT5, we hypothesise that the key to the good performance of the CNN applied within the data assimilation cycling lies in preventing the accumulation of mass in $h$. When mass accumulates in clear regions, i.e. regions where the nature run holds $h<h_{\mathrm{c}}$, it has a snowball effect not only on $h$ itself but also on $r$ (see Fig. 4). After all, clouds, and later rain, are produced whenever $h>h_{\mathrm{c}}$. For dT5, the CNN does not score much better than for dT10 in terms of absolute mass error. However, it was able to effectively remove all bias in $h$ (with a residual of $\mathcal{O}\left(10^{-5}\right)$ ), in contrast to the $\mathrm{CNN}$ for dT10.

To support this claim, we trained an additional CNN with the training set corresponding to $\mathrm{dT}=10$, with a penalty term for the mass of $h$ in the loss function as follows:

$\hat{J}_{\eta}\left(\mathbf{y}_{j}^{\mathrm{p}}(\boldsymbol{w})\right)=J\left(\mathbf{y}_{j}^{\mathrm{p}}(\boldsymbol{w})\right)+\frac{\eta}{n}\left|\sum_{i=1}^{n} y_{j, i, 2}^{\mathrm{p}}-\sum_{i=1}^{n} y_{j, i, 2}\right|$,

where the parameter $\eta$ is tunable. The larger $\eta$, the better the mass of $h$ is conserved at the expense of the RMSE (see Fig. 5). We found a good trade-off for $\eta=2$. We refer to this experiment as $\mathrm{dT} 10_{\eta=2}$. The training process is illustrated in Fig. 5. The mass conservation term comprises about $40 \%$ of the total loss function $\hat{J}$. Both terms of the loss function are decreasing at approximately the same rate throughout the entire training process. Comparing Table 1 with Table 2, we conclude that by adding the penalty term for the mass violation in the loss function, $7 \%$ of the improvement was lost in terms of loss function $J$, but $29 \%$ was gained in the conservation of mass. Table 3 suggests that the CNN is especially active in clear regions or at the edge of clear regions. Indeed, by far the most significant correlations are with $h, r$ and $\frac{\mathrm{d} h}{\mathrm{~d} x}$, 
Table 1. The loss function, the mean RMSE of the variables $u, h$ and $r$, the absolute mass error divided by the number of grid points $n$ for $h$ and $r$, and the bias of $h$ (columns) calculated for the input $\mathbf{X}^{\text {valid }}$ (top row) and the CNN prediction (middle row) with respect to the output $\mathbf{Y}^{\text {valid }}$ for the validation data sets. The last row shows the improvement in the prediction towards the output compared to the input in percentage. The two sections in the table correspond to dT5 and dT10, respectively.

\begin{tabular}{|c|c|c|c|c|c|c|c|c|}
\hline & Validation & Loss & $u$ & $h$ & $r$ & Mass $h$ & Mass $r$ & Bias $h$ \\
\hline \multirow{3}{*}{ dT5 } & Input & $4.9 \times 10^{-2}$ & $4.2 \times 10^{-2}$ & $4.6 \times 10^{-2}$ & $5.9 \times 10^{-2}$ & $1.8 \times 10^{-2}$ & $4.0 \times 10^{-3}$ & $1.4 \times 10^{-2}$ \\
\hline & Prediction & $3.6 \times 10^{-2}$ & $4.1 \times 10^{-2}$ & $3.9 \times 10^{-2}$ & $2.7 \times 10^{-2}$ & $1.4 \times 10^{-2}$ & $2.9 \times 10^{-3}$ & 0.0 \\
\hline & Improvement (\%) & 27 & 2.8 & 15 & 55 & 22 & 28 & 100 \\
\hline \multirow{3}{*}{ dT10 } & Input & $9.6 \times 10^{-2}$ & $7.9 \times 10^{-2}$ & $9.0 \times 10^{-2}$ & $1.2 \times 10^{-1}$ & $3.6 \times 10^{-2}$ & $8.0 \times 10^{-3}$ & $3.3 \times 10^{-2}$ \\
\hline & Prediction & $6.5 \times 10^{-2}$ & $7.3 \times 10^{-2}$ & $6.9 \times 10^{-2}$ & $5.4 \times 10^{-2}$ & $2.9 \times 10^{-2}$ & $7.1 \times 10^{-3}$ & $2.3 \times 10^{-2}$ \\
\hline & Improvement (\%) & 32 & 7.8 & 24 & 55 & 20 & 11 & 30 \\
\hline
\end{tabular}

Table 2. The same as Table 1 but for $\mathrm{dT} 10_{\eta=2}$.

\begin{tabular}{lrrrrrrr}
\hline Validation & Loss & $u$ & $h$ & $r$ & Mass $h$ & Mass $r$ & Bias $h$ \\
\hline Input & $9.6 \times 10^{-2}$ & $7.9 \times 10^{-2}$ & $8.9 \times 10^{-2}$ & $1.2 \times 10^{-1}$ & $3.6 \times 10^{-2}$ & $7.8 \times 10^{-3}$ & $3.3 \times 10^{-2}$ \\
Prediction & $7.2 \times 10^{-2}$ & $7.9 \times 10^{-2}$ & $8.2 \times 10^{-2}$ & $5.5 \times 10^{-2}$ & $1.8 \times 10^{-2}$ & $7.9 \times 10^{-3}$ & $8.9 \times 10^{-4}$ \\
Improvement (\%) & 25 & 0.7 & 8.1 & 53 & 49 & -1 & 103 \\
\hline
\end{tabular}
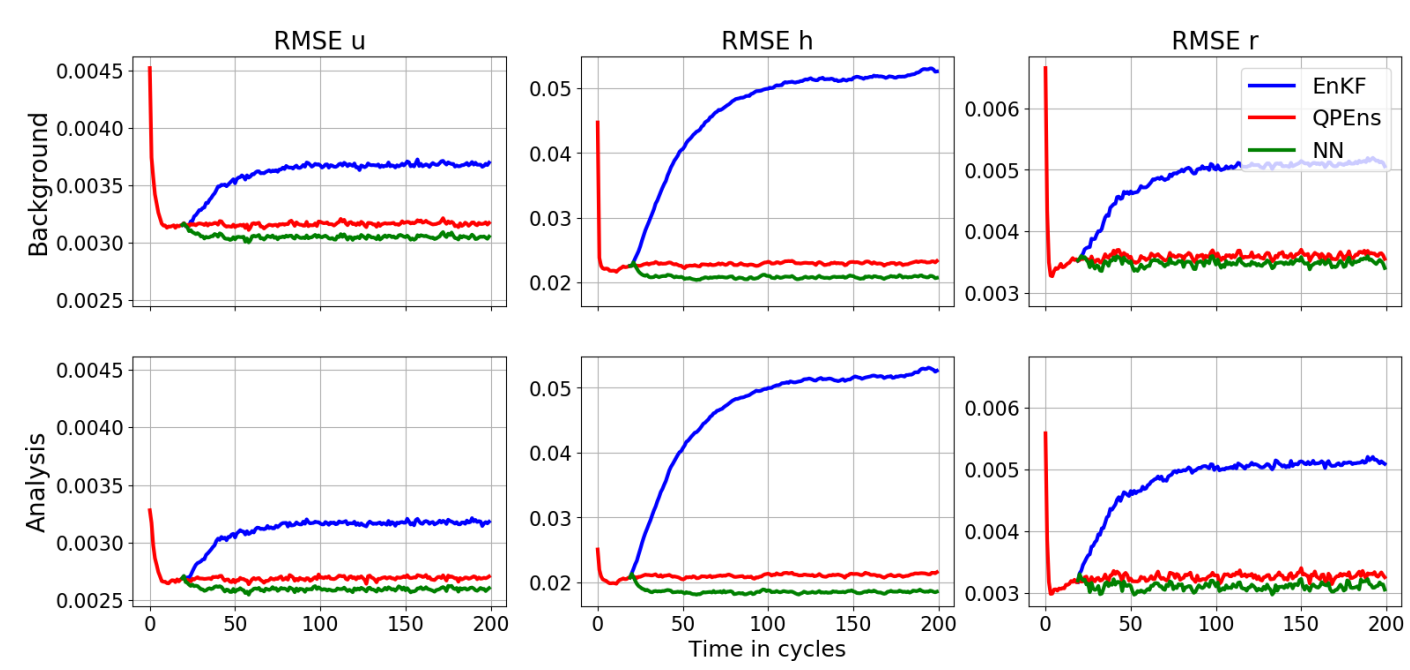

Figure 6. The same as Fig. 3 but for $\mathrm{dT} 10_{\eta=2}$.
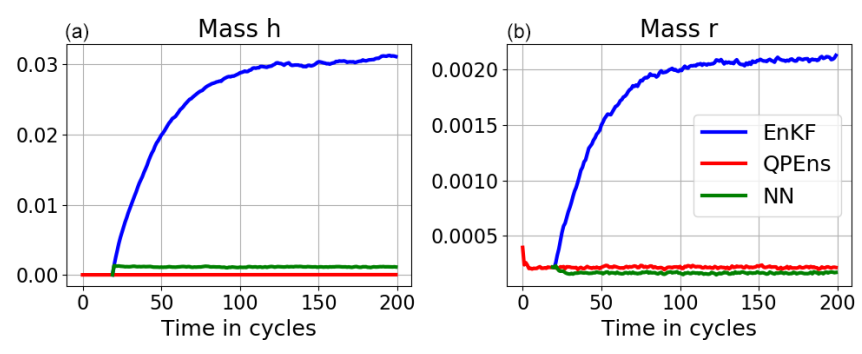

Figure 7. The same as Fig. 4 but for $\mathrm{dT} 10_{\eta=2}$.

where the negative sign indicates that the $\mathrm{CNN}$ corrects more in clear regions than in cloudy regions.
Figures 6, 7 and 8 show the data assimilation results for $\mathrm{dT} 10_{\eta=2}$. It is striking that the CNN performs slightly better than the QPEns. Since the CNN only has an influence radius of five grid points and the localisation cut-off radius of the data assimilation is eight grid points, it is possible that the better results of the CNN stem from this shorter influence radius. However, a $\mathrm{CNN}$ trained on the same data but with kernel sizes of 5 instead of 3 (leading to an influence radius of 10 grid points) yields similar results as in Figs. 6 and 7 (not shown). When comparing the input $\mathbf{X}$, output $\mathbf{Y}$ and the $\mathrm{CNN}$ prediction $\mathbf{Y}^{\mathrm{p}}$ to the nature run, we found that, for the clear regions, $\mathbf{Y}^{\mathrm{p}}$ is slightly closer to the nature run in terms of RMSE than the QPEns and significantly closer than 
Table 3. Correlation coefficient for increments of the output (left column) and the prediction for dT10 (middle column) and dT10 $\eta=2$ (right column) with the input (top row) and the gradient of the input (bottom row).

\begin{tabular}{|c|c|c|c|c|c|c|c|c|c|c|}
\hline & \multicolumn{3}{|c|}{$\mathbf{Y}-\mathbf{X}$} & \multicolumn{3}{|c|}{ dT10: $\mathbf{Y}^{\mathrm{p}}-\mathbf{X}$} & \multicolumn{3}{|c|}{$\mathrm{dT} 10_{\eta=2}: \mathbf{Y}^{\mathrm{p}}-\mathbf{X}$} \\
\hline & & $u$ & $h$ & $r$ & $u$ & $h$ & $r$ & $u$ & $h$ & $r$ \\
\hline \multirow{3}{*}{$\mathbf{X}$} & $u$ & -0.1 & 0.0 & 0.0 & -0.2 & 0.0 & 0.0 & -0.1 & 0.1 & 0.0 \\
\hline & $h$ & 0.0 & -0.1 & -0.1 & 0.1 & -0.2 & -0.2 & 0.2 & -0.5 & -0.2 \\
\hline & $r$ & 0.0 & -0.1 & -0.3 & 0.1 & -0.2 & -0.4 & 0.1 & -0.4 & -0.4 \\
\hline \multirow{3}{*}{$\frac{\mathrm{d} \mathbf{X}}{\mathrm{d} x}$} & $u$ & -0.1 & 0.0 & 0.0 & -0.2 & 0.1 & 0.0 & -0.2 & 0.1 & 0.0 \\
\hline & $h$ & -0.2 & -0.1 & -0.2 & -0.4 & -0.2 & -0.2 & -0.4 & -0.2 & -0.2 \\
\hline & $r$ & -0.1 & -0.1 & -0.3 & -0.2 & -0.2 & -0.3 & -0.2 & -0.1 & -0.3 \\
\hline
\end{tabular}

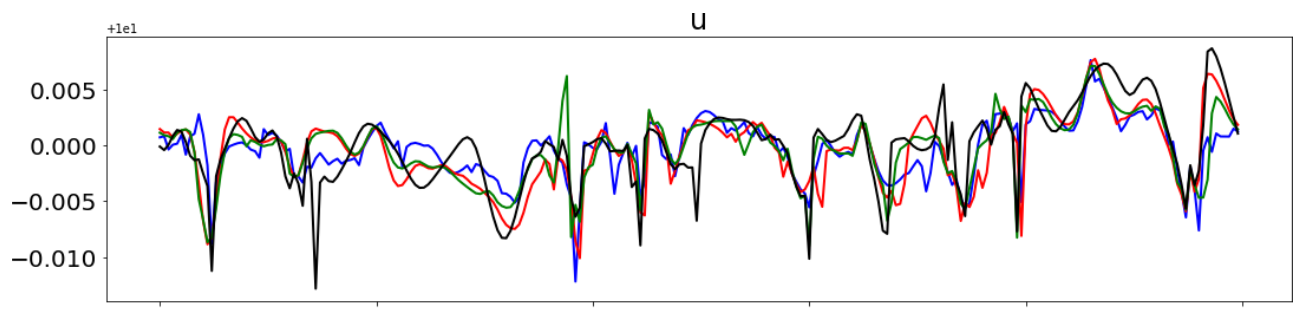

h

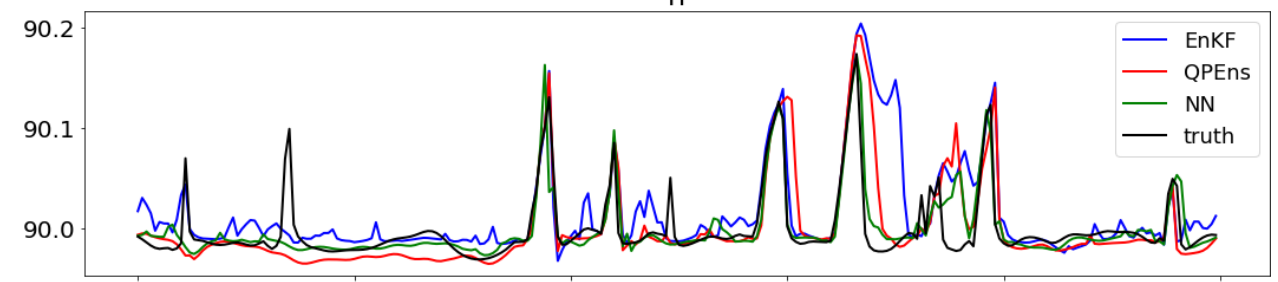

r

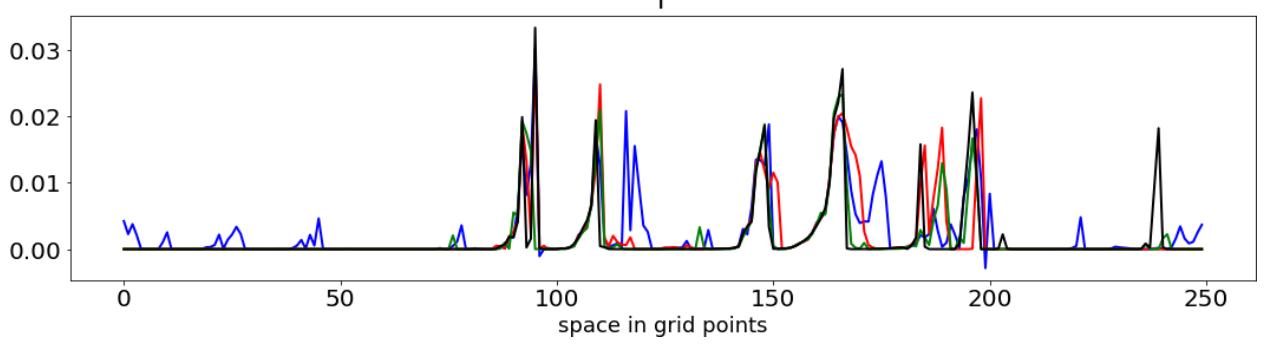

Figure 8. Truth (black) and ensemble mean snapshot for EnKF (blue), QPEns (red) and NN with dT10 $\eta=2$ (green) before negative rain values are set to zero for the EnKF.

the EnKF (not shown). We speculate that this is because the QPEns generally lacks mass in regions where there are no clouds in both the nature run and the QPEns estimate. The EnKF, on the other hand, overestimates the mass in these regions. This is clearly visible in the snapshot of Fig. 8. As a result, the true value of $h$ lies between the QPEns and EnKF estimates. In these regions, it is therefore favourable that the CNN cannot completely close the gap between the input and output data, as it leads to a better fit to the nature run. We also performed an experiment where $h$ is updated by the $\mathrm{CNN}$, and the other variables remain equal to the EnKF solution, and similar results were obtained as in Figs. 6 and 7. When only the clear regions of $h$ are updated by the CNN, the positive influence of the CNN is slightly reduced, but it still matches the performance of the QPEns. We, therefore, conclude that the success of this approach lies in the ability of the CNN to correct for errors of $h$, especially in clear regions.

\section{Conclusion}

Geoscience phenomena have several aspects that are different from standard data science applications, for example, governing physical laws, noisy observations that are nonuniform in space and time from many different sources and rare, interesting events. This makes the use of NNs particu- 
larly challenging for convective-scale applications, although attempts have been made for predicting rain, hail or tornadoes (McGovern et al., 2019). The approach taken in this study combines noisy and sparse observations with a dynamical model using a data assimilation algorithm and, in addition, uses a CNN to improve on the conservation of physical laws. In previous work it was shown that, in idealised set-ups, conserving physical quantities like mass in the data assimilation framework using the QPEns can significantly improve the estimate of the nature run. Here we show that it is possible to obtain similar positive results by training a CNN to conserve mass in a weak sense. By training on the unconstrained (EnKF)/constrained (QPEns) input/output pair, the $\mathrm{CNN}$ is able to reduce the mass violation significantly. Moreover, we found that adding a penalty term for mass violation in the loss function is necessary in one of the two test cases to produce data assimilation results that are as good as those corresponding to the QPEns.

These encouraging results prompt the question of the feasibility of this approach being applied to fully complex numerical weather prediction systems. The challenge here lies in the generation of the training data. First, the effectiveness of conserving different quantities has to be verified in a nonidealised numerical weather prediction framework, where the quantities to be conserved may not be known and may not be exactly conserved within the numerical weather prediction model (Dubinkina, 2018). A second consideration is the computational cost. Advances are made in this regard (Janjic et al., 2021) but effort and collaboration with optimisation experts are still required to allow the generation of a reasonably large training data set.

Code and data availability. The provided source code (https://doi.org/10.5281/zenodo.4354602, Ruckstuhl et al., 2020) includes the necessary scripts to produce the data.

Author contributions. YR set up and performed the experiments and prepared the paper with contributions from all coauthors. SR set up the code for the CNN. TJ contributed to the scientific design of the study and the analysis of the numerical results.

Competing interests. The authors declare that they have no conflict of interest.

Financial support. This research has been supported by the German Research Foundation (DFG; subproject B6 of the Transregional Collaborative Research Project SFB/TRR 165, "Waves to Weather" and grant no. JA 1077/4-1).

Review statement. This paper was edited by Alberto Carrassi and reviewed by Marc Bocquet and Svetlana Dubinkina.

\section{References}

Bishop, C. H., Etherton, B. J., and Majumdar, S.: Adaptive sampling with the ensemble transform Kalman filter. Part I: Theoretical aspects., Mon. Weather Rev., 129, 420-436, 2001.

Bocquet, M., Brajard, J., Carrassi, A., and Bertino, L.: Bayesian inference of chaotic dynamics by merging data assimilation, machine learning and expectation-maximization, Foundations of Data Science, 2, 55-80, https://doi.org/10.3934/fods.2020004, 2020.

Brajard, J., Carrassi, A., Bocquet, M., and Bertino, L.: Combining data assimilation and machine learning to emulate a dynamical model from sparse and noisy observations: A case study with the Lorenz 96 model, J. Comput. Sci.-Neth, 44, 101171, https://doi.org/10.1016/j.jocs.2020.101171, 2020a.

Brajard, J., Carrassi, A., Bocquet, M., and Bertino, L.: Combining data assimilation and machine learning to infer unresolved scale parametrisation, arXiv [preprint], arXiv:2009.04318, 9 September 2020 b.

Brenowitz, N. D. and Bretherton, C. S.: Spatially Extended Tests of a Neural Network Parametrization Trained by Coarse-Graining, J. Adv. Model. Earth Sy., 11, 2728-2744, https://doi.org/10.1029/2019MS001711, 2019.

Burgers, G., van Leeuwen, P. J., and Evensen, G.: Analysis Scheme in the Ensemble Kalman Filter., Mon. Weather Rev., 126, 17191724, 1998.

Chollet, F.: Deep Learning with Python, Manning Publications Company, Greenwich, CT, USA, 2017.

Cintra, R. S. C. and de Campos Velho, H. F.: Data Assimilation by Artificial Neural Networks for an Atmospheric General Circulation Model: Conventional Observation, CoRR, abs/1407.4360, arXiv [preprint], arXiv:1407.4360, 16 July 2014.

Dubinkina, S.: Relevance of conservative numerical schemes for an Ensemble Kalman Filter, Q. J. Roy. Meteor. Soc., 144, 468-477, https://doi.org/10.1002/qj.3219, 2018.

Dueben, P. D. and Bauer, P.: Challenges and design choices for global weather and climate models based on machine learning, Geosci. Model Dev., 11, 3999-4009, https://doi.org/10.5194/gmd-11-3999-2018, 2018.

Evensen, G.: Sequential data assimilation with a nonliear quasigepstrophic model using Monte Carlo methods to forecast error statistics., J. Geophys. Res., 99, 10143-10162, 1994.

Evensen, G.: Data Assimilation: The Ensemble Kalman Filter, Springer-Verlag Berlin Heidelberg, Germany, 2009.

Farchi, A., Laloyaux, P., Bonavita, M., and Bocquet, M.: Using machine learning to correct model error in data assimilation and forecast applications, arXiv [preprint], arXiv:2010.12605, 23 October 2020.

Gaspari, G. and Cohn, S. E.: Construction of correlation functions in two and three dimensions, Q. J. Roy. Meteor. Soc., 125, 723757, 1999.

Haslehner, M., Janjic, T., and Craig, G. C.: Testing particle filters on simple convective-scale models. Part 2: A modified shallow-water model, Q. J. Roy. Meteor. Soc., 142, 1628-1646, https://doi.org/10.1002/qj.2757, 2016.

Hunt, B. R., Kostelich, E. J., and Szunyogh, I.: Efficient Data Assimilation for Spatiotemporal Chaos: A local Ensemble Transform Kalman filter., Physica, 230, 112-126, 2007.

Janjić, T., McLaughlin, D., Cohn, S. E., and Verlaan, M.: Conservation of mass and preservation of positivity with ensemble-type 
Kalman filter algorithms, Mon. Weather Rev., 142, 755-773, 2014.

Janjic, T., Ruckstuhl, Y., and Toint, P. L.: A data assimilation algorithm for predicting rain, Q. J. Roy. Meteor. Soc., accepted with minor revisions, 2021.

Jin, J., Lin, H. X., Segers, A., Xie, Y., and Heemink, A.: Machine learning for observation bias correction with application to dust storm data assimilation, Atmos. Chem. Phys., 19, 10009-10026, https://doi.org/10.5194/acp-19-10009-2019, 2019.

Klambauer, G., Unterthiner, T., Mayr, A., and Hochreiter, S.: Self-Normalizing Neural Networks, arXiv [preprint], arXiv:1706.02515, 8 June 2017.

Krasnopolsky, V. M., Fox-Rabinovitz, M. S., and Belochitski, A. A.: Using ensemble of neural networks to learn stochastic convection parameterizations for climate and numerical weather prediction models from data simulated by a cloud resolving model, Adv. Artif. Neural Syst., 2013, 485913, https://doi.org/10.1155/2013/485913, 2013.

LeCun Yann, Bengio Yoshua, and Hinton Geoffrey: Deep learning, Nature, 521, 436-444, https://doi.org/10.1038/nature14539, 2015.

McGovern, A., Elmore, K. L., Gagne, David John, I., Haupt, S. E., Karstens, C. D., Lagerquist, R., Smith, T., and Williams, J. K.: Using Artificial Intelligence to Improve Real-Time DecisionMaking for High-Impact Weather, B. Am. Meteor. Soc., 98, 2073-2090, https://doi.org/10.1175/BAMS-D-16-0123.1, 2017.

McGovern, A., Lagerquist, R., John Gagne, David, I., Jergensen, G. E., Elmore, K. L., Homeyer, C. R., and Smith, T.: Making the Black Box More Transparent: Understanding the Physical Implications of Machine Learning, B. Am. Meteor. Soc., 100, 2175 2199, https://doi.org/10.1175/BAMS-D-18-0195.1, 2019.

Moosavi, A., Attia, A., and Sandu, A.: Tuning Covariance Localization Using Machine Learning, in: Computational Science, ICCS 2019. Lecture Notes in Computer Science, vol. 11539, Springer, Cham., https://doi.org/10.1007/978-3-030-22747-0_16, 2019.

Nielsen, M. A.: Neural networks and deep learning, Determination press, available at: https://books.google.ch/books?id= STDBswEACAAJ (last access: 2 February 2021), 2015.

Pathak, J., Hunt, B., Girvan, M., Lu, Z., and Ott, E.: ModelFree Prediction of Large Spatiotemporally Chaotic Systems from Data: A Reservoir Computing Approach, Phys. Rev. Lett., 120, 024102, https://doi.org/10.1103/PhysRevLett.120.024102, 2018a.

Pathak, J., Wikner, A., Fussell, R., Chandra, S., Hunt, B. R., Girvan, M., and Ott, E.: Hybrid forecasting of chaotic processes: Using machine learning in conjunction with a knowledge-based model, Chaos: An Interdisciplinary J. Nonlinear Sci., 28, 041101, https://doi.org/10.1063/1.5028373, 2018 b.

Rasp, S. and Lerch, S.: Neural networks for post-processing ensemble weather forecasts, Mon. Weather Rev., 146, 3885-3900, https://doi.org/10.1175/MWR-D-18-0187.1, 2021.
Rasp, S. and Thuerey, N.: Data-driven medium-range weather prediction with a Resnet pretrained on climate simulations: A new model for WeatherBench, arXiv [preprint], arXiv:2008.08626, 19 August 2020.

Rasp, S., Pritchard, M. S., and Gentine, P.: Deep learning to represent subgrid processes in climate models, P. Natl. Acad. Sci., 115, 9684-9689, https://doi.org/10.1073/pnas.1810286115, 2018.

Rasp, S., Dueben, P. D., Scher, S., Weyn, J. A., Mouatadid, S., and Thuerey, N.: WeatherBench: A benchmark dataset for data-driven weather forecasting, J. Adv. Model. Earth Syst., 12, e2020MS002203, https://doi.org/10.1029/2020MS002203, 2020.

Reichstein, M., Camps-Valls, G., and Stevens, B.: Deep learning and process understanding for data-driven Earth system science, Nature, 566, 195-204, https://doi.org/10.1038/s41586019-0912-1, 2019.

Ruckstuhl, Y. and Janjić, T.: Parameter and state estimation with ensemble Kalman filter based algorithms for convectivescale applications, Q. J. Roy. Meteor. Soc, 144, 826-841, https://doi.org/10.1002/qj.3257, 2018.

Ruckstuhl, Y., Rasp, S., Würsch, M., and Janjic, T.: $\mathrm{CNN}$ to conserve mass in data assimilation, Zeonodo, https://doi.org/10.5281/zenodo.4354602, 2020.

Scher, S. and Messori, G.: Weather and climate forecasting with neural networks: using general circulation models (GCMs) with different complexity as a study ground, Geosci. Model Dev., 12, 2797-2809, https://doi.org/10.5194/gmd-12-2797-2019, 2019.

Watson, P. A. G.: Applying Machine Learning to Improve Simulations of a Chaotic Dynamical System Using Empirical Error Correction, J. Adv. Model. Earth Sy., 11, 1402-1417, https://doi.org/10.1029/2018MS001597, 2019.

Weyn, J. A., Durran, D. R., and Caruana, R.: Improving datadriven global weather prediction using deep convolutional neural networks on a cubed sphere, J. Adv. Model. Earth Syst., 12, e2020MS002109, https://doi.org/10.1029/2020MS002109, 2020.

Würsch, M. and Craig, G. C.: A simple dynamical model of cumulus convection for data assimilation research, Meteorol. Z., 23, 483-490, 2014.

Yuval, J. and O'Gorman, P. A.: Stable machine-learning parameterization of subgrid processes for climate modeling at a range of resolutions, Nat. Commun., 11, 1-10, 2020.

Zeng, Y. and Janjić, T.: Study of conservation laws with the Local Ensemble Transform Kalman Filter, Q. J. Roy. Meteor. Soc., 142, 2359-2372, https://doi.org/10.1002/qj.2829, 2016.

Zeng, Y., Janjić, T., Ruckstuhl, Y., and Verlaan, M.: Ensembletype Kalman filter algorithm conserving mass, total energy and enstrophy, Q. J. Roy. Meteor. Soc., 143, 2902-2914, https://doi.org/10.1002/qj.3142, 2017. 